\title{
Biomecánica fémoro-patelar
}

\author{
J. USABIAGA ZARRANZ; R. CUELLAS GUTIERREZ; \\ R. CRESPO ROMERO Y G. DE LA HERRÁN NÚÑEZ \\ Servicio de Traumatología y Cirugía Ortopédica. \\ Hospital Nuestra Señora de Aranzazu (Osakidetza). San Sebastián
}

\section{APLICACION CLINICA DE LA BIOMECANICA FEMOROPATELAR}

La articulación femoro-patelar es un diseño biomecánico original. La rótula, pese a sus pequeñas dimensiones, está destinada a transmitir las mayores presiones que se producen en el aparato locomotor, imprescindibles para que el individuo corra, salte, etc.

Lo que más llama la atención en cuanto a su diseño es que a pesar de las extraordinarias presiones que debe soportar, la congruencia entre rótula y fémur es muy variable y cambia:

- De unos tipos a otros según el tipo morfológico de rótula.

- En el mismo sujeto según el grado de flexión en que se encuentre la rodilla.

Además de ello, y a pesar de las grandes presiones que debe soportar, nunca llega a utilizar toda su superficie articular disponible de contacto.

\section{FUNCIONES DE LA RÓTULA:}

Para Ficat ${ }^{8}$ y Hungerford ${ }^{13}$ la función más importante de la rótula es el trabajo que desarrolla, en el movimiento de extensión de la rodilla, consiguiendo aumentar la distancia del aparato extensor al eje de movimiento de la articulación femoro-tibial en la flexo-extensión. Esta separación consigue un aumento de fuerza cercano al $50 \% 8$.

Por otro lado evita la fricción del tendón cuadricipital con la tróclea al separar el aparato extensor del fémur. El coeficiente de fricción cartílago-cartílago es menor que el del tejido fibroso-cartílago. El tejido fibroso soporta bien tracciones pero no fricción. El tejido cartilaginoso tolera bien la presión y la fricción.

En este trabajo cuenta con la ayuda del líquido sinovial, que en conjunto con la superficie cartílago-cartílago constituye el mejor método de fricción. El líquido sinovial es el lubricante ideal y cuando experimentalmente se ha sustituido por algún otro, el coeficiente de fricción aumenta ${ }^{21}$. Solamente la fricción por cojinetes de aire, últimamente empleada en la industria, presenta unos coeficientes de fricción mejores que este sistema biológico.

Por otro lado, la patela actúa como una guía centrando la rótula en la tróclea y evitando que se luxe. Actúa como una polea de fricción ${ }^{17}$. También tiene un papel de defensa en traumatismos directos por el efecto amortiguador del cartílago. Finalmente tiene una función estética en la morfología de la rodilla.

\section{PRESIONES FEMORO-PATELARES}

A lo largo del movimiento de flexión y de una manera simplista, la presión femoro-patelar sería la resultantes de las fuerzas ejercidas por la tracción del tendón cuadricipital y por la tensión del tendón rotuliano. Esta resultante es perpendicular a la superficie articular en cada momento. Casi todos los autores recurren a este paralelogramo para sus estudios 1,5,8,13,14,15,16 (figura 1).

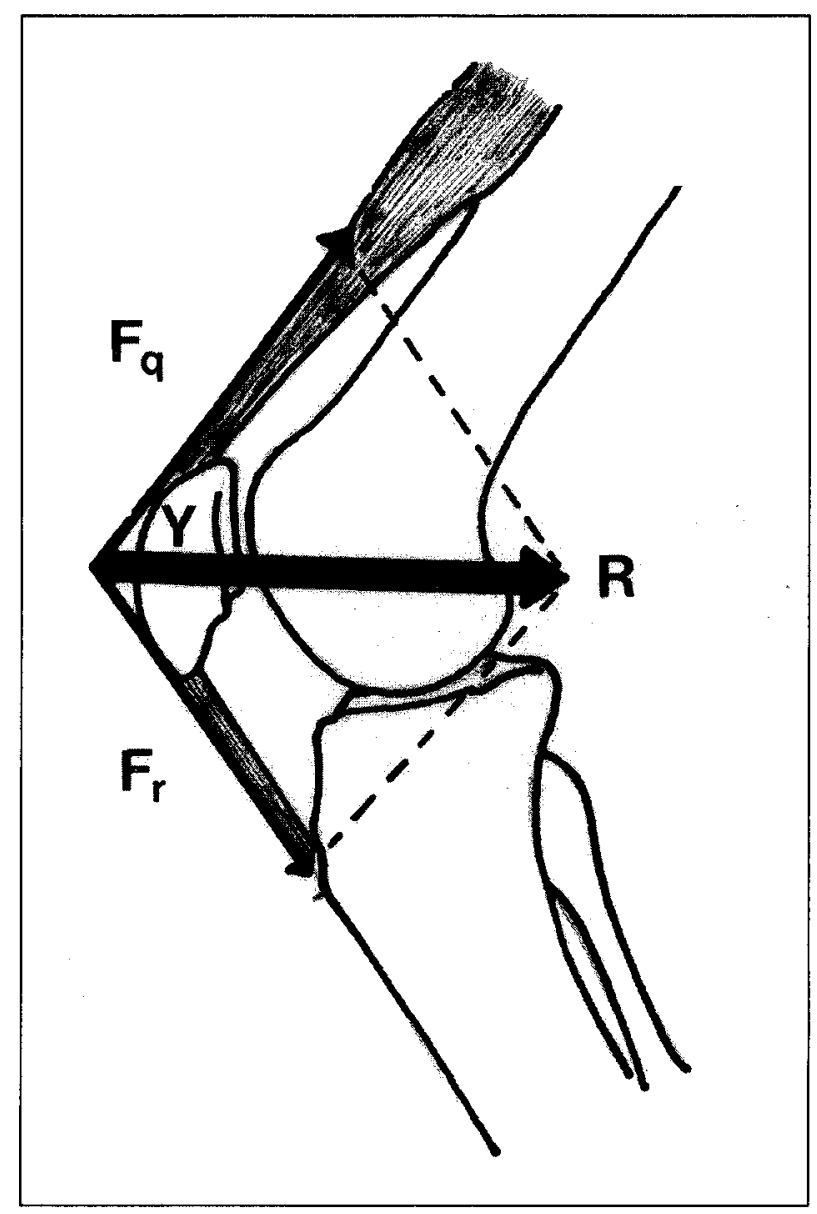

Figura 1: Fuerza de compresión fémoro-patelar. 
Muchos factores influyen en la intensidad de esta fuerza: En primer lugar el grado de flexión, que al cerrar el ángulo, hace aumentar la resultante. Además influye el peso del individuo. Sea cual fuere éste, al agacharse un individuo, el peso, perpendicular al suelo desde el centro de gravedad del cuerpo, queda por detrás de la rodilla y debe ser compensado por la tensión de los dos tendones de anclaje rotuliano que evitarán que el individuo se desplome hacia atrás.

La resultante de compresión femoro-rotuliana es pues muy variable dependiendo de las diferentes posiciones y actividades de la rodilla. En general a mayor flexión, el peso del cuerpo está más desplazado hacia atrás, actuando así con un brazo de palanca mayor. Para compensarlo, la tracción del cuadríceps debe ser mucho mayor y por tanto se incrementará la presión femoropatelar para mantener el equilibrio. La resultante de estos dos factores: peso y grado de flexión, hace que se multiplique el efecto compresivo sobre la femoro-patelar (figura 2).

Como ayuda a la articulación femoro-patelar en este trabajo, el individuo normalmente flexiona la cadera hacia delante, con lo que el centro de gravedad se desplazará en esa dirección pasando más cerca de la articulación de la rodilla y siendo por tanto su resultante o brazo menor. Todos sabemos que es más fácil permanecer en cuclillas inclinado hacia adelante, que con el tronco erguido. El individuo con problemás patelares,

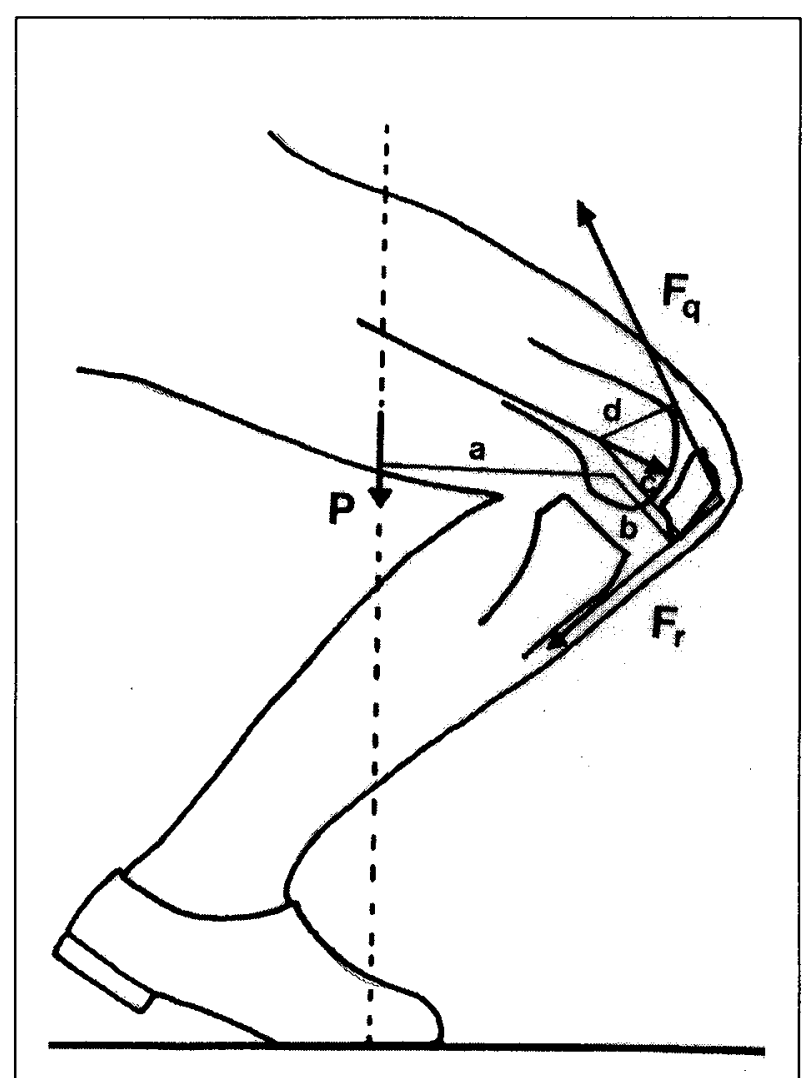

Figura 2. Situación de equilibrio en un sujeto agachado. El peso del cuerpo actúa como un brazo de palanca (a) que debe ser compensado por la tensión del cuadríceps. cuando tenga que flexionar sus rodillas, lo deberá hacer inclinando el tronco hacia delante (figura 3).

Con la flexión además el centro de giro de la articulación se va desplazando hacia atrás, y ello hace que aún deba aumentar más la tensión del cuadríceps para mantener el equilibrio.

\section{VALORACIÓN DE LAS PRESIONES FEMORO-PATELARES}

Por lo que se refiere al cálculo o cuantificación de las presiones femoro-patelares, diferentes autores las han estudiado con diferentes métodos y con resultados dis-

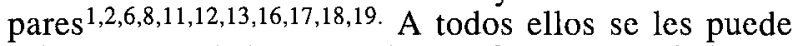
achacar el no haber contado con factores asociados en los movimientos cotidianos como son aceleración, deceleración, inercia, tensiones cápsulo-ligamentosas, potencia muscular, etc. También influye mucho la morfología rotuliana. De cualquier forma el que la rótula soporta grandes presiones nos lo demuestra el hecho de que la naturaleza la haya dotado del cartílago hialino más grueso del organismo.

El primer intento de valoración de la presión femoropatelar lo realizó Fick en el año 1.904 según referencia de Henche ${ }^{11}$. Desde entonces, muchos autores lo han intentado obteniendo cifras que varían desde los 150 $\mathrm{Kg}$. para un individuo de $80 \mathrm{~kg}$. al descender escalcras, hasta los más de $1.000 \mathrm{~kg}$. reseñados por otros autores ${ }^{20}$. Las cifras más elevadas las refieren los autores que utilizan métodos vectoriales o teóricos en sus determinaciones. Siendo menores cuando la determinación se hace por métodos directos en especial a grados altos de flexión. 1,6,7,11,12,16,19,20 Esto hace suponer que la articulación posee mecanismos de defensa para disminuir las presiones.

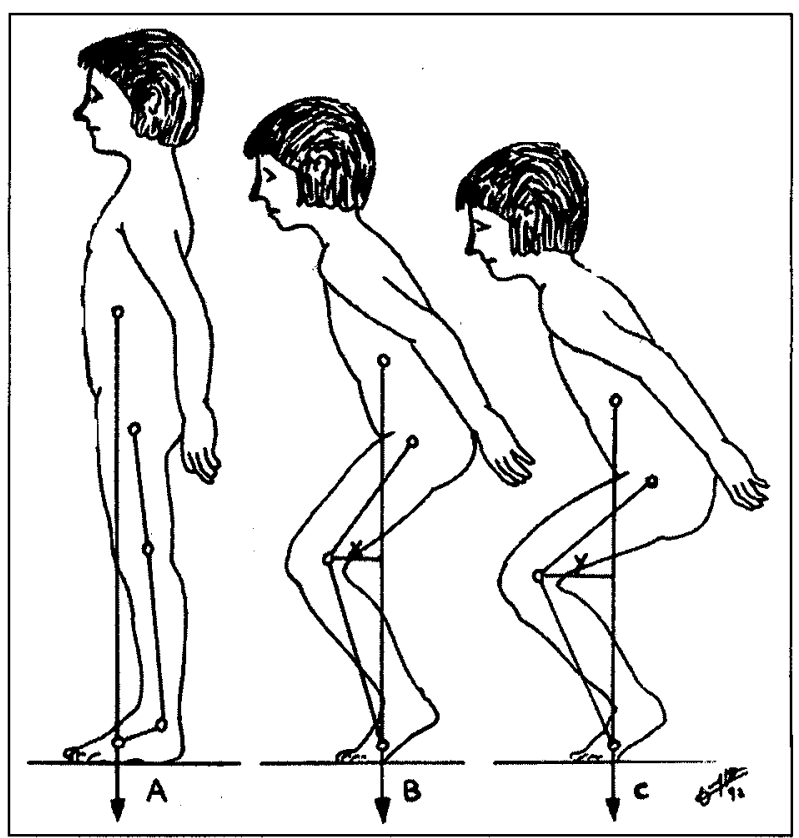

Figura 3: Variación del brazo de palanca con que actúa el peso del cuerpo al ahacharse e inclinar el tronco hacia delante. 


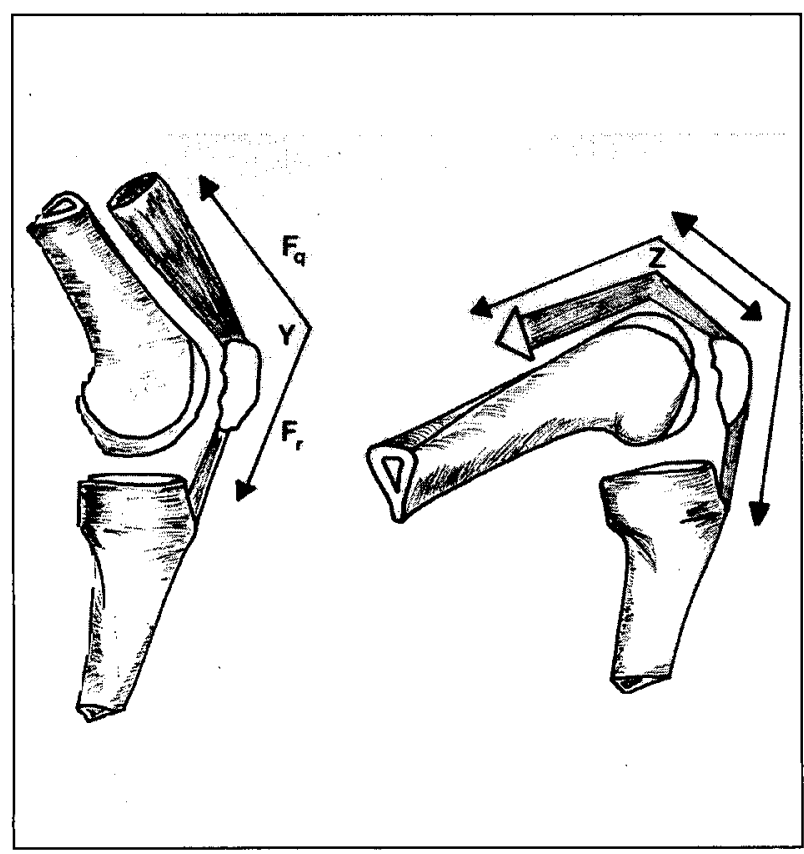

Figura 4: Mecanismo de doble polea por el que a partir de los $90^{\circ}$ de flexión se produce un contacto tendón-fémur que impide que se cierre más el ángulo, y consecuentemente favorece la descompresión femoro-patelar.

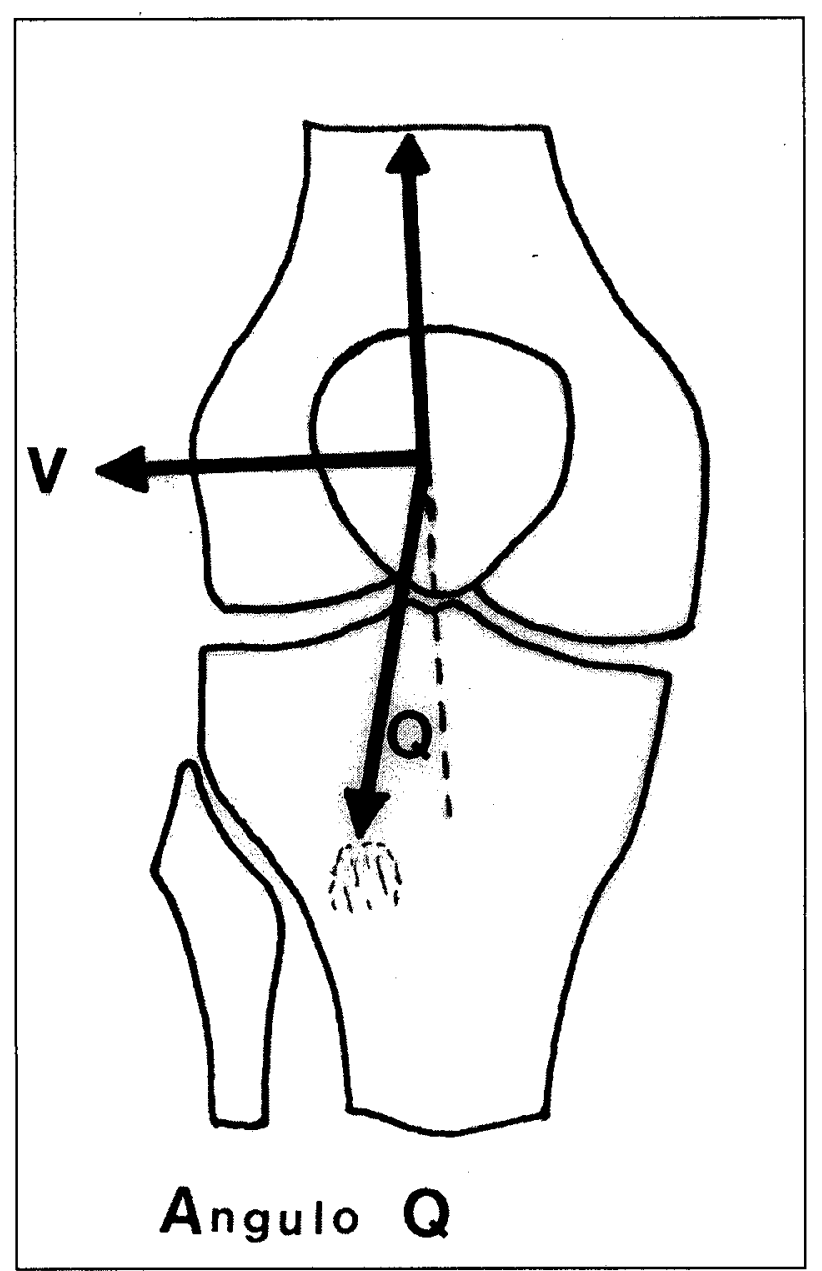

Figura 5: Angulo $Q$ en extensión. Lo forman el valgo fisiológico y el atornillamiento tibial. Vector lateral de valgo.
Uno de estos mecanismos de defensa lo constituye la puesta en marcha de una segunda polea, constituida por el tendón cuadricipital que descarga a la articulación femoro-patelar (figura 4). Este sistema es más importante en pacientes con patela baja. En esta situación el tendón soporta parte de las presiones. A $120^{\circ}$ de flexión soporta casi un tercio de la presión teórica femoro-patelar. En pacientes patelectomizados además de la pérdida del elemento cartilaginoso de fricción se pierde este efecto de doble polea.

Blaimont $^{2}$ determina que si la articulación femoropatelar soportase los valores obtenidos teóricamente, la rótula se partiría; por lo que es evidente con la ayuda de otros elementos fisiológicos (efecto de segunda polea, contracciones musculares, etc.) que las descargan de su función.

Dependiendo del grado de flexión, la fuerza del cuadríceps varía y con algunas actividades, como subir escaleras, la presión puede llegar a ser 3,3 veces el peso corporal, equivalente a unas siete veces la presión que soporta al caminar. ${ }^{18}$ A ello se debe el dolor desencadenado al subir escaleras en pacientes con lesiones femoro-patelares.

Matthews ${ }^{17}$ determina que la articulación femorotibial comparada con la femoro-patelar soporta mayores presiones en las actividades normales, pero cuenta con la ayuda de los meniscos. Sin embargo, a altos grados de flexión, observa como la femoro-patelar soporta solicitaciones mucho mayores.

Un dato a tener en cuenta cuando se revisan estos trabajos es que la presión se reparte por una superficie que nunca es la rótula completa por lo que el parámetro presión/ $\mathrm{cm}^{2}$ será mayor de las cifras aportadas, siendo este parámetro el real. Además de ello, estos cálculos se han realizado para actividades de tipo sedentario que se verán multiplicadas en la práctica deportiva.

Lo que si está claro es que en esta situación de grandes presiones, cualquier alteración de ejes conlleva una mala distribución de las presiones que afectaría rápidamente al cartílago. Probablemente sea el factor más importante en la aparición de lesiones. Sabemos que la artrosis proviene de una presión articular muy elevada o transmitida en una dirección incorrecta sobre el cartílago, de tal manera que se sobrepasa la resistencia de éste.

\section{DINÁMICA ROTULIANA}

En los últimos grados de extensión de la rodilla la tibia rota externamente en relación al fémur. Con ello se lateraliza la tuberosidad anterior de la tibia en el plano frontal. Es lo que constituye el llamado mecanismo de atornillamiento.

Esta rotación, unida al valgo físiológico del aparato extensor, hace que se incremente el ángulo entre la dirección del cuadríceps y la del tendón rotuliano. Es el llamado ángulo $\mathrm{Q}$ de Brattstrom y que va a ser determinante en las solicitaciones externas que va a sufrir la rótula. Este ángulo Q aumenta en extensión y disminuye al flexionar la rótula (figura 5). 


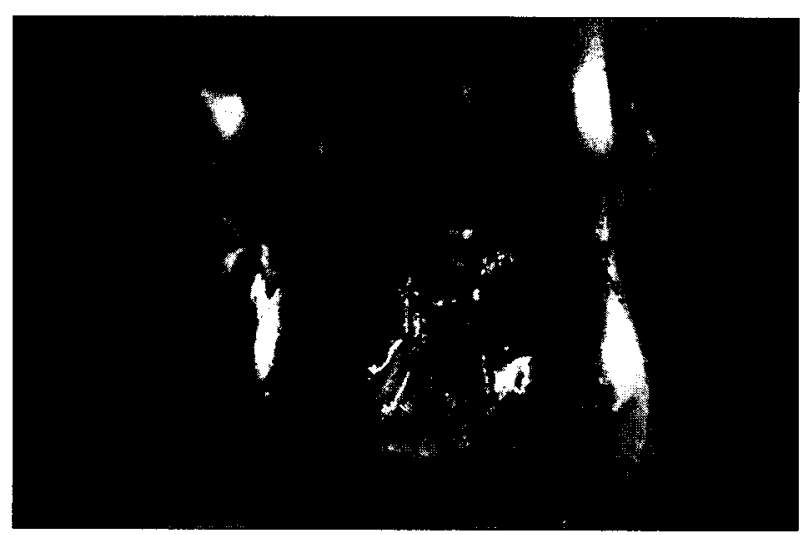

Figura 6: Rotación en el eje cráneo-caudal de la rótula con la flexo-extensión. Derecha: El contacto femoro-patelar a partir de los $90^{\circ}$ se realiza en vertiente externa y «old facet» con los cóndilos. Izquierda: Al extender el contacto es vertiente interna y externa quedando libre la «old facet».

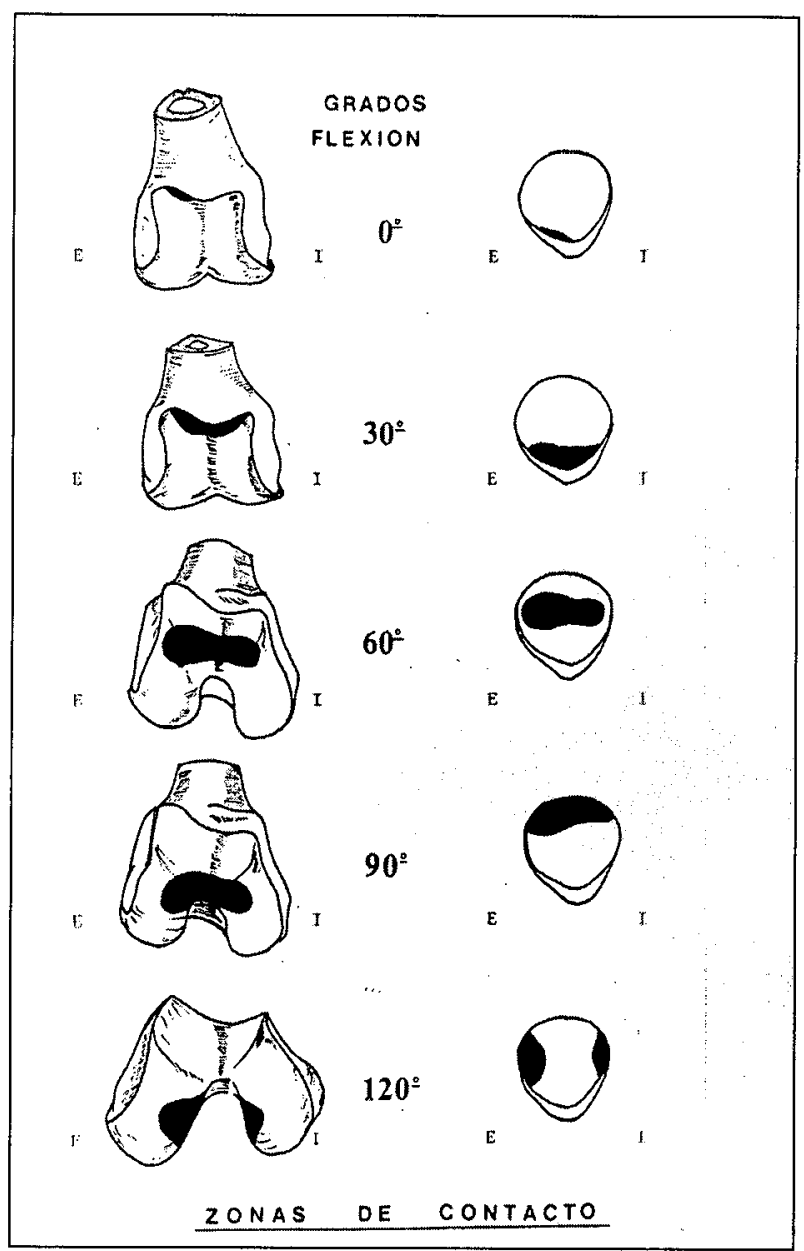

Figura 7: Zonas de contacto femoro-patelares correspondientes a cada grado de flexión.
$\mathrm{El}$ ángulo $\mathrm{Q}$ determina el vector de valgo que tiende a desplazar la rótula hacia fuera. En extensión o cerca de ella la rótula tenderá a salirse de la tróclea. Serán los alerones y la tensión del vastro interno los que la sujetan.

En los primeros $20^{\circ}$ de flexión la tibia desrota, decreciendo el ángulo Q. La rótula entra en la tróclea femoral tomando contacto con ella por la vertiente externa, ya que la tróclea es más alta proximalmente en dicha vertiente. A medida que progresa la flexión la rótula encaja en la tróclea, la estabilidad es mayor y será el cóndilo externo el que evite su salida. DelgadoMartins ${ }^{4}$ fué el primero que estudió la dinámica de la rótula con o sin contracción del cuadríceps utilizando $\mathrm{TAC}$ en estos primeros grados de flexión, no visibles en radiología convencional. Hoy en día con técnicas de TC tridimensional podrán estudiarse más a fondo estos movimientos.

A los $90^{\circ}$ de flexión, la rótula tiende a lateralizarse y da aspecto de cubrir más al cóndilo externo que al interno. A partir de aquí sufre un pequeño movimiento de rotación sobre su eje longitudinal tendiendo la carilla interna a hacerse más posterior. Este movimiento similar al «tilting» patológico, pero en este caso fisiológico y de sentido contrario, cambia la función de la carrilla interna. Esta carrilla se encaja en la escotadura intercondílea, entrando a tomar contacto con el cóndilo interno la tercera carilla u «old facet» (figura 6). Por ello se crean grandes presiones en la cresta que separa ambas, ya que no es congruente con la superficie que contacta, motivo por el que aparecen lesiones en esta zona. ${ }^{9}$

Así pues, a grados bajos de flexión, el contacto se hace tróclea-vertientes quedando libre la «old facet». A altos grados el contacto en la parte interna se hace entre esta tercera carilla y el cóndilo interno quedando libre la vertiente que quedará encajada en la escotadura.

En su conjunto la rótula, en el plano sagital, realiza un movimiento de traslacción circunferencial sobre un radio fijo que es la longitud del tendón rotuliano. En este plano se ve trasladada hacia posterior con la flexión de la rodilla.

\section{ÀREA DE CONTACTO FÉMORO-PATELAR}

Muchos autores lo han estudiado coincidiendo casi todos ellos. Los métodos de estudio han sido muy diversos. $3,6,7,8,9,10,11,12,17$

La distribución de la superficie de contacto va trasladándose hacia proximal en la cara articular de la rótula a medida que progresa la flexión (figura 7). El contacto inicial es en el polo inferior y aparece entre los $0^{\circ}$ y los $20^{\circ}$ de flexión, dependiendo de la longitud del tendón rotuliano, por lo que las patelas bajas toman contacto antes. A partir de aquí la superficie se desplaza hacia arriba hasta llegar a los $90^{\circ}$ en que contacta el polo superior. A partir de aquí el contacto se realiza por dos superficies separadas que corresponden a la «old facet» y a una parte de la vertiente externa. En estos últimos grados también hay contacto entre tendón cuadricipital y tróclea femoral, como ya hemos descrito (figura 4). 
Por lo que respecta a la superficie de contacto en trocleacóndilos, la superficie se desplaza en sentido inverso. Inicia el contacto en la parte más superior y externa de la tróclea para posteriormente ir desplazándose por la tróclea hacia abajo. A los $90^{\circ}$ llega a la escotadura intercondílea trasladándose luego el contacto a ambos cóndilos.

En general casi todos los autores están de acuerdo en que a mayor grado de flexión el área superficie de contacto femoro-patelar aumenta.

\section{BIBLIOGRAFÍA}

1. Bandi W, Brennwald J. The significance of femoropatellar pressure in the pathogenesis and treatment of chondromalacia patellae and femoropatellar arthrosis. En «The Knee Joint». Ingwersen. New York. American Alsevier Publishing Co. Inc. 1.974 .

2. Blaimont $P$, Van Elegem $P$, Alameh $M$, Klein $P$, Auquier $O$, Halleux P. Contribution à l'étude des contraintes patellaires. Hypothèse pathogénique de 1'arthrose fémoro-patellaire. Acta Orthop Belg. 1.983. 49 Fasc. 437-445.

3. Burnotte J, Jourdain M, Blaimont $P$, Fairen $M$, Halleux $P$. Contributions a l'étude des contraintes fémoro-patellaires. Etude des surfaces de contact fémoro-patellaires au cours de la flexion du genu. Acta Orthop Belg. 1.976. 42 Sppl. 1. 144-152.

4. Delgado-Martins H. A study of the position of the patella using Computerised tomography. J Bone Jt Surg. 1.979. 61-B. 443444.

5. Denham R.A, Bishop R.E.D. Mechanics of the Knee and problems in reconstructive Surgery. J Bone Jt Surg. 1.978. 60-B. 345-352.

6. Ferguson A.B, Brown T, Fu F, Rutkowski R. Relief de patellofemoral contac stress by anterior displacement of the tibial tubercle. J Bone Jt Surg. 1.979. 61-A. 159-166.
7. Ferrandez L, Usabiaga J, Yubero J, Sagarra J, De No L. An experimental study of the redistribution of patellofemoral pressures by the anterior displacement of the anterior tuberosity of the tibia. Clin Orthop. 1.989. 238. 183-189.

8. Ficat P, Hungerford D.S. Disorders of the patello-femoral joint Ed. Másson. Baltimore. USA. 1.977.

9. Goodfellow J, Hungerford D.S, Zindel M. Patello-femoral joint mechanics and pathology. Functional anatomy of the patellofemoral joint. J Bone Jt Surg. 1.976. 58-B. 2287-290.

10. Goymann V, Muller H.G. New calculation of the biomechanics of the patello-femoral joint and its clinical significance. En «The Knee Joint». International Congress Series. 324. Amsterdam. Excepta Medica. 1.974.

11. Henche H.R, Kunzi H.U, Morscher E. The areas of contact pressure inthe patello-femoral joint. International Orthopaedics (SICOT). Springer Verlag. 1.981. 4. 279-281.

12. Huberti H.H, Hayes W.C. Patello-femoral contact pressures. J Bone Jt Surg. 1.984. 55-A. 715-724.

13. Hungerford D.S, Barry M. Biomechanics of the patello-femoral joint. Clin Orthop. 1.979. 144. 4.

14. Maquet P.G. Mechanics and osteoarthritis of $y$ the patello-femoral joint. Clin Orthop. 1.979. 144. 70-73.

15. Maquet P.G. Rappe] biomécanique. Symposium 54 reunion SOFCOT. Deséquilibres et chondropathies de la rotule. Paris. 1.979. Rev Chir Orthop. 1.980. 66. 209-211

16. Maquet P.G. Biomechanics of the Knee. Springer Verlag. Berlin. 1.984.

17. Matthews L.S, Sonstegard D.A, Henke J.A. Load bearing characteristics of the patello-femoral joint. Acta Orthop Scand. 1.977. 48. 511-516.

18. Reilly D.T, Martens M. Experimental analysis of the quadriceps muscle force and patello-femoral joint reacction force for various activities. Acta orthop Scand. 1.972. 43. 126-137.

19. Retaillaud J.L, Darmana R, Devallet P, Mansat M, Morucci J.P. Etude biomécanique expérimentale de l'avancement de la tubérosité tibiale. Rev Chir Orthop. 1.989. 75. 513-523.

20. Seedhom B.B, Tsubuku M. A technique for the study of contact between visco-elastic bodies with special reference to the patello-femoral joint. J Biomechanics. 1.977. 104. 253-260.

21. Swanson S.A.V. Lubrification dans les articulations synoviales. Acta Orthop Belg. 1.973. 39 Suppl. 1. 33-42 